\title{
Using Simulation to Improve Medical Students' Comfort with Selected Pediatric Procedures
}

\author{
Mark E Harrison, M.D., Deborah A Kroeker, M.D., Carolyn R Ahlers-Schmidt, Ph.D. \\ University of Kansas School of Medicine-Wichita \\ Department of Pediatrics
}

\begin{abstract}
Background. Simulation in pediatrics is described often in more procedurally-heavy areas, such as in intensive care, emergency medicine, and neonatology. However, there is a paucity of literature related to simulation in general pediatrics. We sought to improve students' comfort with and knowledge about selected procedures using simulation mannequins during their pediatric rotation.

Methods. During a workshop, third year medical students received a lecture on male circumcisions, lumbar punctures, the Ortolani and Barlow maneuvers, and ear examinations. Following the lecture, the students were given hands-on instruction and feedback on the techniques for performing ear and hip exams, lumbar punctures, and circumcisions. Students took a pre- and post-encounter assessment regarding their confidence level, procedural knowledge, and perceived usefulness of the training. Wilcoxon Signed Rank tests were used to determine changes in the students' confidence levels and knowledge. Alpha was set at 0.05 for all comparisons.
\end{abstract}

Results. Sixty medical students (100\%) participated in the study during the 2012/2013 academic year. Confidence and knowledge increased significantly on all procedures following the simulation experience $(p<0.001)$. Perception of usefulness of the training also increased significantly at post-test $(\mathrm{p}=0.019)$.

Conclusion. Medical students benefited from using simulation to demonstrate and practice common pediatric procedures, both in their confidence and knowledge. The use of simulation for general pediatric procedures should improve patient safety, as well as remove some of the anxiety of performing procedures in actual clinical scenarios.

KS J Med 2015; 8(2):72-79.

\section{Introduction}

Medical students frequently voice concerns regarding their comfort level in performing some of the most basic and essential procedures encountered during their pediatric rotation. A well-proven way to increase learners' skills and comfort is through the use of simulations, ${ }^{1-3}$ which improve patient safety ${ }^{4}$ and decrease medical student anxiety when performing a procedure for the first time in an actual clinical situation. ${ }^{5,6}$
Third year medical students on their pediatrics rotations have varied exposure to and comfort levels with infants and children as patients. There are many challenges facing students as they approach three of the most common procedures: ear and hip exams, lumbar punctures, and circumcisions. ${ }^{7}$

Ear/hip exams. The ear and hip examinations can be difficult with real moving (and often crying) patients. It is helpful to practice looking through the ear canal to the tympanic membrane of a 
mannequin prior to looking in the ear canal of a child with an ear infection. Looking at pictures of ear pathology in textbooks or discussing what a dislocated infant hip feels like does not substitute for hands-on experience.

Lumbar puncture. Students may have had previous experience performing lumbar punctures on adult patients, but unique skills are needed for success with the infant patient. $^{8}$ Among pediatric interns, only onethird have performed a lumbar puncture on an infant, fewer than $75 \%$ have observed the procedure, and confidence and competency uniformly are rated low. ${ }^{9-11}$ Despite this, entering interns perform this procedure frequently and early in their training programs.

Circumcision. Students would benefit from a simulation experience prior to performing a circumcision on an actual patient, particularly when they may feel like they are being observed closely by a parent. Simulation models for circumcision are a relatively recent development, but early research suggests that they increase the learner's comfort with the procedure. ${ }^{12}$ Additionally, some medical students do not have the opportunity to perform a circumcision on a newborn, so they benefit from learning to perform a circumcision in a simulated experience.

The purposes of simulation include training and practicing procedures in a safe environment and assessing procedural skills. It is used at all levels, throughout the traditional medical school years, ${ }^{13,14}$ as well as in graduate medical education. ${ }^{5,15-17}$ As more interest has developed in interprofessional learning, the use of simulation has spread to other areas of medical education as well. ${ }^{18-20}$ A PubMed search for articles published in the last five years related to the use of simulation in medical education returned hundreds of articles. Simulation in pediatrics is described often in the more procedurally-heavy areas such as intensive care, emergency medicine, and neonatology. ${ }^{4,21}$ However, there is a scarcity of literature related to the use of simulation to teach medical students to perform the most common procedures in pediatrics. To determine if simulation also can be used to increase medical student comfort in basic examination and procedural skills, a simulation session was added to the medical student curriculum.

\section{Methods}

Every six weeks, a new group of six to eight medical students rotated through the pediatrics department at our university. Part of one afternoon of this rotation was dedicated to learning to perform circumcisions, lumbar punctures, Ortolani and Barlow maneuvers, and ear examinations in a hands-on, experiential format. Students were instructed in the correct techniques for circumcisions and lumbar punctures and practiced these procedures on the models following instruction. Pediatricians were present to guide the medical students through the procedures and to offer immediate feedback on their technique. Additionally, students were instructed in proper examination techniques of neonates to assess for developmental dysplasia of the hips using the Ortolani and Barlow maneuvers. Students then practiced these maneuvers on an infant mannequin. Finally, the students were instructed in the correct techniques to examine a patient's tympanic membrane and ear canal and were instructed in some techniques for positioning infants/toddlers to facilitate an easier and safer ear exam. Teaching included information on the diagnosis of tympanic membrane and ear canal pathology, aided by ear models with varying pathologies. Following the instruction, students practiced using the ear model. 
Measurements. The students took a preand post-training assessment regarding confidence level and procedural knowledge. Short-answer questions regarding each procedure were used to evaluate knowledge and scored for accuracy by a physician. The experience was measured using a 6-point Likert scale $(1=$ not at all confident, $6=$ very confident) to determine their level of confidence in their skills in circumcision, lumbar punctures, the Ortolani and Barlow maneuvers, and ear examinations, both before and after the simulations. A similar scale was used to assess the students' anticipated and perceived usefulness of the simulation experience. Finally, students were allowed to provide written feedback at the end of the post-assessment.

Participation in the research portion of the training (i.e., completing the assessment) was optional. Surveys were numbered to allow matching of pre-post surveys, while allowing responses to remain anonymous.

Statistical analysis. The Wilcoxon Signed Rank tests were used to contrast the pre- and post-experience to determine the change in the students' confidence levels and knowledge regarding the performance of these procedures. Data were summarized by dichotomizing the Likert-scale at the fifth level ("Confident") to ascertain how many students felt confident or very confident before and after the simulation. Both anticipated and perceived benefit of training were averaged for evaluation, and feedback responses were reviewed and summarized. Alpha was set at 0.05 for all comparisons.
The study was approved by the university's Human Subjects Committee.

\section{Results}

Sixty medical students (100\%) participated in the simulation training and the research study during the 2012/2013 academic year. University demographics in this class included 35 (58\%) males with a mean age of 28 years ( $\mathrm{SD}=3$ years). The majority were Caucasian (48; 80\%) followed by African American/Black (3; $5 \%)$, Hispanic (3; 5\%), and other (5; 8\%). One participant did not identify a race/ethnicity (2\%).

Prior to the simulation training, student confidence in the procedures was low for circumcision (median 1, IQR 1-2.75), lumbar puncture (median 1, IQR 1-2), and hip exam (median 2, IQR 1.25-4). Students were more confident with ear exams at baseline (median 4, IQR 3-4). Student confidence increased significantly on all procedures following the simulation experience ( $p<0.001$; Figure 1$)$. The highest levels of confidence after the experience were reported for ear (median 5, IQR 4-5) and hip exams (median 5, IQRs 4-6). Only one student $(2 \%)$ reported a decrease in confidence performing the Ortolani or Barlow maneuver, with a confidence level of 6 (highest level) on the pretest and a 5 on the posttest. On evaluation of qualitative feedback, this student reported that the infant hip mannequin was too rigid and was not an accurate representation of real infant hips. 


\section{Confidence Outcomes}

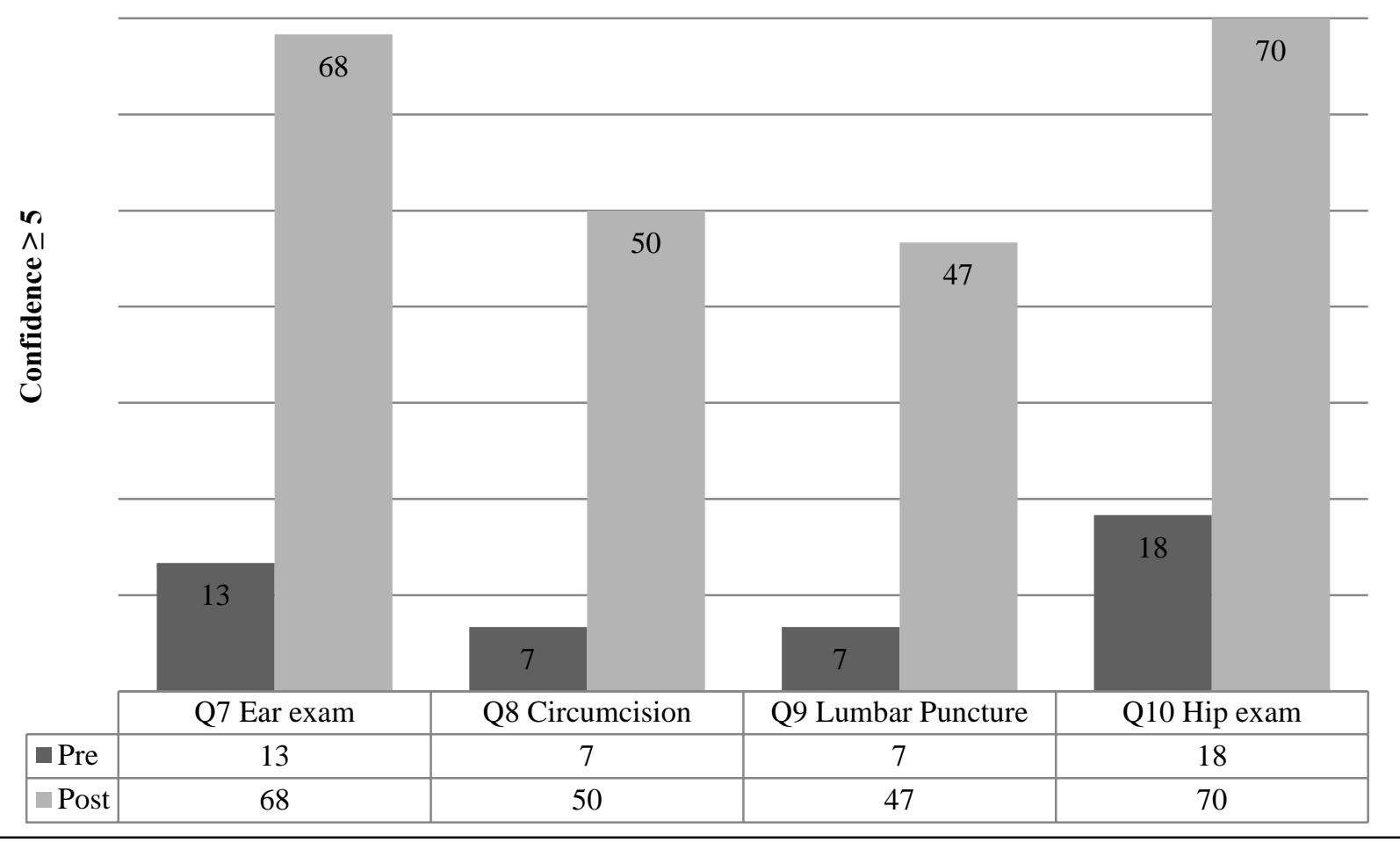

Figure 1. Confidence outcomes pre- and post-simulation training.

Knowledge measured before the simulation training indicated fewer than $50 \%$ of students were able to answer four out of six items. Greater than $50 \%$ of students were able to identify risks of both circumcision and lumbar puncture. Only three students (5\%) were able to answer all six knowledge questions correctly prior to the training. Student knowledge increased significantly following the experience $(\mathrm{z}=$ -
6.977, $\mathrm{p}<0.001$; Figure 2). On postexamination, 44 students (73.3\%) correctly answered all six knowledge questions. Greater than $80 \%$ of students responded correctly on each of the items after the training. Inexplicably, one student (2\%) had a lower score on the post knowledge assessment, with four items correct on the pre-test and only two correct on the posttest. 


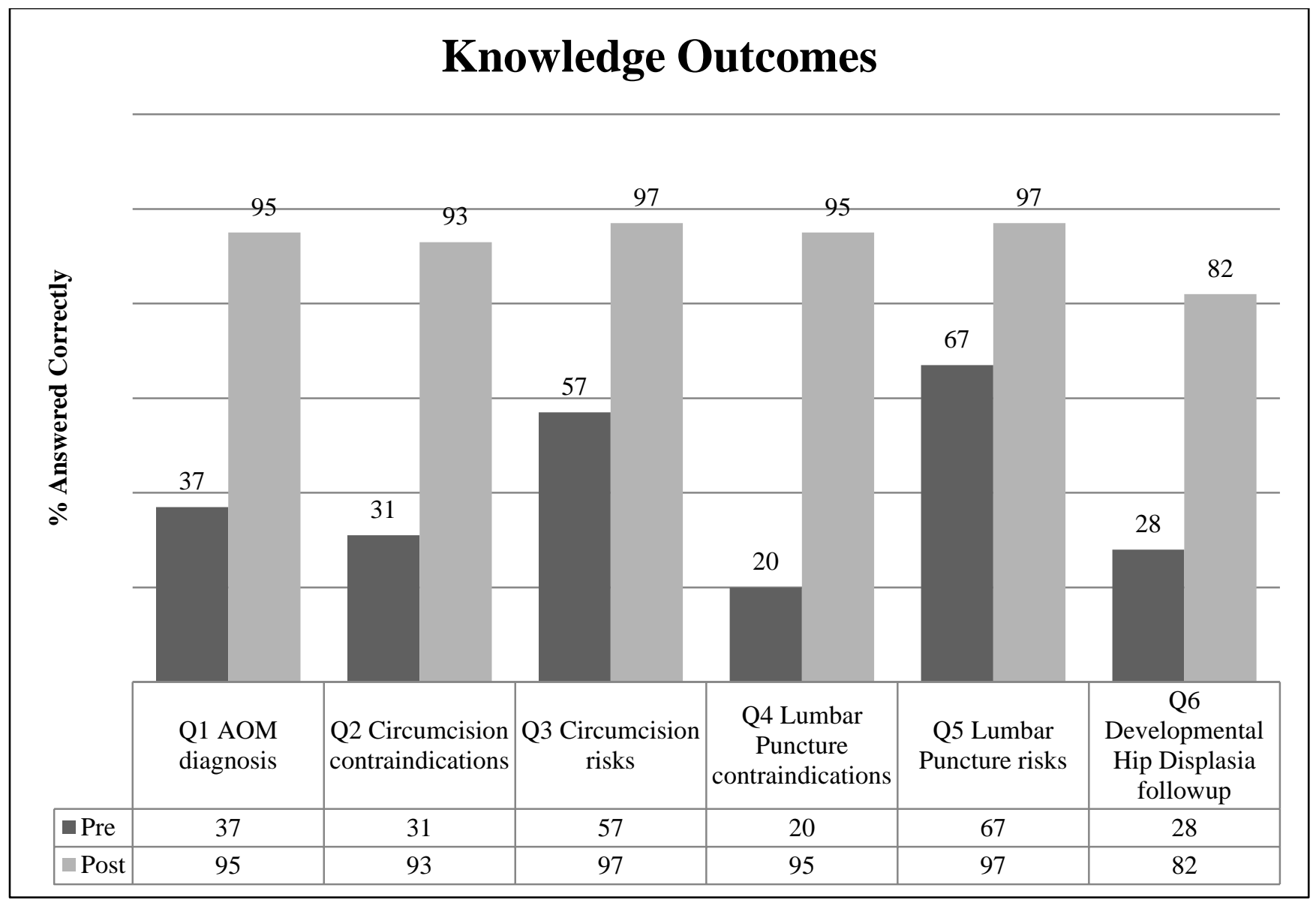

Figure 2. Knowledge outcomes pre- and post-simulation training.

Fifty-nine students (98\%) reported "useful" or "very useful" when asked about perceived benefit of the training. Twenty-six students left qualitative feedback. Among the qualitative responses, 22 (85\%) expressed gratitude for the training or described it as "helpful" and/or "useful" and three $(12 \%)$ expressed a desire for more practice. Two students provided constructive criticism: the firmness of the hip mannequin (above) and a recommendation to use fellow students as models for ear exams. The anticipated benefit of training was high (87\% identified it as "useful" or "very useful") at pre-test, but still increased significantly after training $(\mathrm{Z}=-2.353, \mathrm{p}=$ 0.019 ) with $98.3 \%$ of subjects rating the training as "useful" or "very useful" at posttest (Figure 3). 


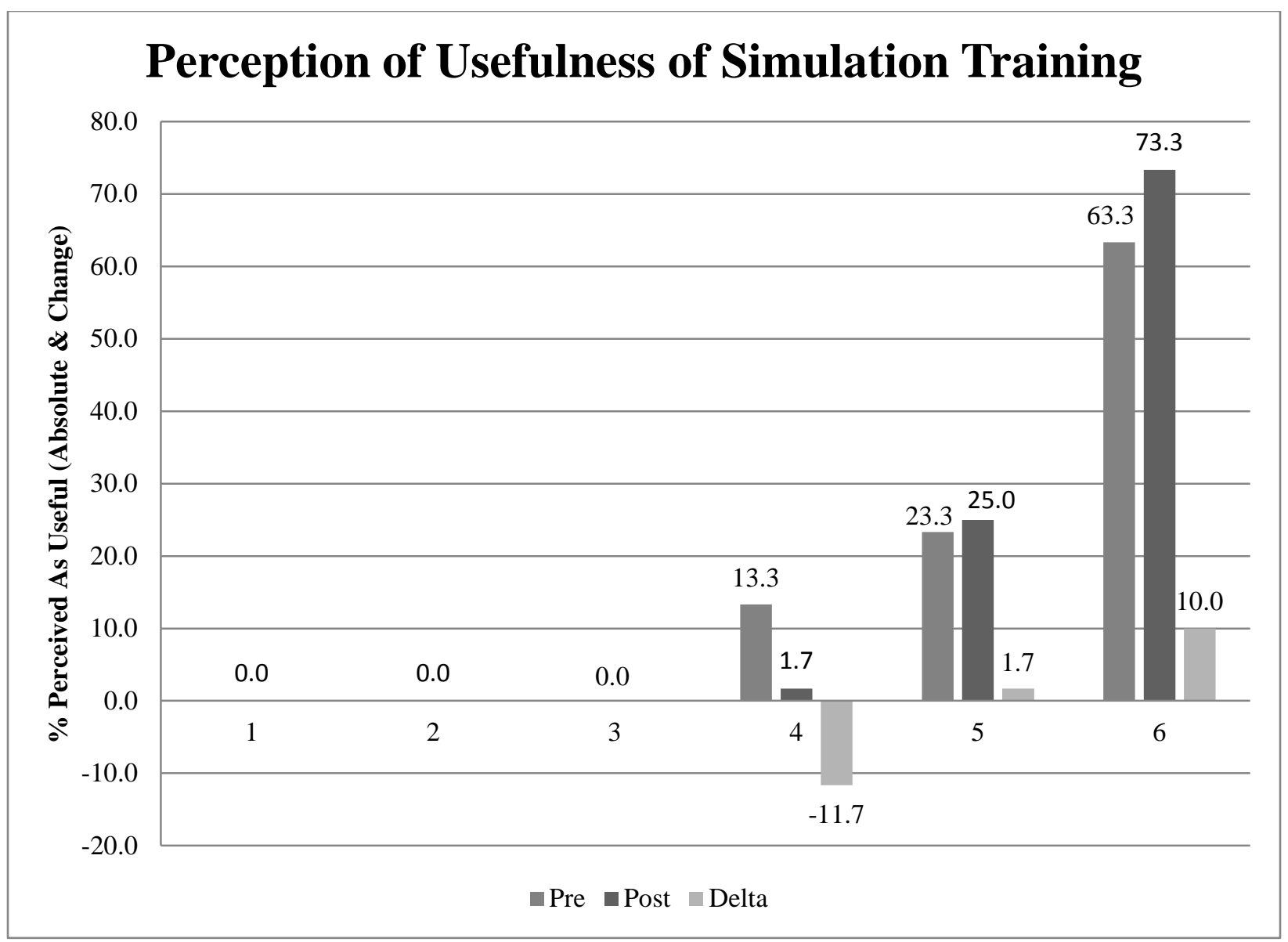

Figure 3. Students' perception of usefulness of activity.

\section{Discussion}

These results showed that medical students' knowledge and confidence grew through participating in the simulation experience. The medical students valued the opportunity to learn the proper techniques to perform these procedures. In spite of high perceived value at pre-test, a significant increase was present at post-test. In addition, student comments indicated the learning opportunity was valuable. The reason one medical student ranked his/her confidence in performing the Ortolani and Barlow maneuvers lower after the simulation may be because the mannequin used was quite stiff, making it more difficult than it is in real life to assess for a positive examination. However, the purpose of the simulation is to teach the general principles of how to perform the examination, while allowing the learners to perfect the skill when they are in the clinics and wards. Future simulation mannequins may benefit through exploration of more analogous materials and more lifelike feel, especially with regards to flexibility.

Limitations of this study included that students were not evaluated on their ability to perform the techniques in simulated environments or on actual patients following the simulation and that no comparison group was included. There is a wide field of evidence already examining both correlations between procedural confidence and ability as well as simulation training and procedural competency.

A major strength was participation of the entire cohort in the study. In addition, consistency of trends in the data as both knowledge and confidence increased 
significantly for all procedures suggests a reliability of the measures. The medical students believed they benefited from this experience, gaining both knowledge regarding the procedures and confidence in their ability to perform these procedures. The use of simulation to teach general pediatric procedures is a helpful tool to allow medical students to learn in a safe environment. In an era in which patient safety is paramount, having learners practice procedures on a mannequin is a relief for many educators so they can identify how well a learner can perform a procedure prior to performing it on an actual patient. Similarly, it is often a relief for learners gaining experience in a low-risk setting prior

\section{References}

${ }^{1}$ Grant DJ, Marriage SC. Training using medical simulation. Arch Dis Child 2012; 97(3):255-259. PMID: 21920872.

2 Lenchus JD. End of the "see one, do one, teach one" era: The next generation of invasive bedside procedural instruction. J Am Osteopath Assoc 2010; 110(6):340346. PMID: 20606241.

${ }^{3}$ Weinberg ER, Auerbach MA, Shah NB. The use of simulation for pediatric training and assessment. Curr Opin Pediatr 2009; 21(3):282-287. PMID: 19381090.

${ }^{4}$ Lemoine JB, Daigle SC: Neonatal resuscitation simulation: Improving safety while enhancing confidence and competence. Nurs Womens Health 2010; 14(2):143-145. PMID: 20409138.

${ }^{5}$ van Schaik SM, Von Kohorn I, O'Sullivan P. Pediatric resident confidence in resuscitation skills relates to mock code experience. Clin Pediatr (Phila) 2008; 47(8):777-783. PMID: 18474898.

${ }^{6}$ Burlacu CL, Chin C. Effect of pediatric simulation training on candidate's confidence. Paediatr Anaesth 2008; 18(6):566-567. PMID: 18445209. to performing procedures on real patients, particularly with the possibility of the added clinical stress of having parents stand over them while they perform a procedure for the first time. While this study was not designed to evaluate changes in performance, future studies could supplement this work by evaluation with standardized patient scenarios to students using a pre-post design and/or a control group of solely didactic learners. Further research should be done to assess the long-term impact of this type of simulation training, both on the learners' success in a pediatric residency program and further into the future when they are practicing as medical providers.

${ }^{7}$ Gaies MG, Landrigan CP, Hafler JP, Sandora TJ. Assessing procedural skills training in pediatric residency programs. Pediatrics 2007; 120(4):715-722. PMID: 17908757.

${ }^{8}$ Kessler DO, Auerbach M, Pusic M, Tunik MG, Foltin JC. A randomized trial of simulation-based deliberate practice for infant lumbar puncture skills. Simul Healthc 2011; 6(4):197-203. PMID: 21527870.

9 Auerbach M, Chang TP, Reid J, et al. Are pediatric interns prepared to perform infant lumbar punctures? A multiinstitutional descriptive study. Pediatr Emerg Care 2013; 29(4):453-457. PMID: 23528505.

${ }^{10}$ Pruden CM, Kerrey BT, Mittiga M, Del Rey JG. Procedural readiness of pediatric interns: Defining novice performance through simulation. J Grad Med Educ 2010; 2(4):513-517. PMID: 22132270.

${ }^{11}$ Augustine EM, Kahana M. Effect of procedure simulation workshops on resident procedural confidence and competence. J Grad Med Educ 2012; 4(4):479-485. PMID: 24294425. 
${ }^{12}$ Roca P, Alvarado C, Stausmire JM, Farooq S, Hill-Engstler EA. Effectiveness of a simulated training model for procedural skill demonstration in neonatal circumcision. Simul Healthc 2012; 7(6):362-373. PMID: 23086514.

${ }^{13}$ Ortiz N, Pedrogo Y, Bonet N. Integration of high-fidelity simulator in third-year paediatrics clerkship. Clin Teach 2011; 8(2):105-108. PMID: 21585670.

${ }^{14}$ Tofil NM, Benner KW, Worthington MA, Zinkan L, White ML. Use of simulation to enhance learning in a pediatric elective. Am J Pharm Educ 2010; 74(2):21. PMID: 20414434.

${ }^{15}$ Burwinkle T, Gibson J, Pliego J, Wick L, Coker N, Pohl J. Simulation training in a pediatric residency program. Tex Med 2008; 104(1):50-53. PMID: 18396773.

${ }^{16}$ Gerard JM, Thomas SM, Germino KW, Street MH, Burch W, Scalzo AJ. The effect of simulation training on PALS skills among family medicine residents. Fam Med 2011; 43(6):392-399. PMID: 21656393.

${ }^{17}$ Southgate WM, Annibale DJ. Simulation training in graduate medical education: A means of traversing a changed and changing landscape. Adv Neonatal Care 2010; 10(5):261-268. PMID: 20838077.

${ }^{18}$ Stewart M, Kennedy N, Cuene-Grandidier $\mathrm{H}$ : Undergraduate interprofessional education using high-fidelity paediatric simulation. Clin Teach 2010; 7(2):90-96. PMID: 21134155.

${ }^{19}$ Messmer PR. Enhancing nurse-physician collaboration using pediatric simulation. J Contin Educ Nurs 2008; 39(7):319-327. PMID: 18649808.

${ }^{20}$ Falcone RA Jr, Daugherty M, Schweer L, Patterson M, Brown RL, Garcia VF. Multidisciplinary pediatric trauma team training using high-fidelity trauma simulation. J Pediatr Surg 2008; 43(6):1065-1071. PMID: 18558184.

${ }^{21}$ Anderson JM, Warren JB. Using simulation to enhance the acquisition and retention of clinical skills in neonatology. Semin Perinatol 2011; 35(2):59-67. PMID: 21440812.

Keywords: anatomic models, medical education, pediatrics 